\title{
Deshabituación de benzodiazepinas en un paciente preparando cápsulas de diazepam en dosis decrecientes
}

\author{
Ana Martín Ruiz', Manuel Lozano Bosch ${ }^{2}$, Eva Ferrer Bosch ${ }^{3}$ \\ 1. Licenciada en Farmacia. Farmacia Manuel Lozano Bosch (Valencia). 2. Licenciado en Farmacia. Farmacia Manuel Lozano Bosch (Valencia). 3. Doctora \\ en Farmacia. Farmacia Manuel Lozano Bosch (Valencia).
}

\section{PALABRAS CLAVE}

Benzodiazepinas, farmacia comunitaria, deshabituación

\section{ABREVIATURAS}

BZD: benzodiazepina DDED: dosis diaria equivalente de diazepam

\section{KEYWORDS}

Benzodiazepines, community pharmacy, uninhabited

\section{Presentación del caso/Antecedentes}

El consumo de benzodiazepinas (BZD) ha crecido ininterrumpidamente en España desde los años 90. La mitad de los pacientes mayores de 85 años, y el $2 \%$ de los adolescentes entre 15 y 19 años han estado tratados con BZD (1). Ninguna indicación clínica justifica la prescripción prolongada de BZD; sin embargo, su uso prolongado es muy frecuente (2).

La paciente estaba en tratamiento con alprazolam desde hace 14 años. Durante un año, redujo la dosis partiendo comprimidos de alprazolam $0,5 \mathrm{mg}$ hasta llegar a una pauta de 0,25/0/0,25. Quería dejar la medicación y acudió a su médico para que le prescribiera alguna alternativa.

El tratamiento para la deshabituación de benzodiazepinas (BZD) es la disminución progresiva de la dosis, pero la no existencia de presentaciones comercializadas con las dosis necesarias complica la implementación del tratamiento. El médico nos consulta si podíamos hacer algo al respecto.

\section{Estudio y evaluación}

La paciente tenía una dependencia física y psíquica provocada por el uso prolongado del medicamento y cumplía con los criterios diagnósticos de dependencia. El riesgo de dependencia se incrementa con la dosis utilizada y el tiempo de uso y es mayor cuanto menor es la vida media de la BZD. Antecedentes de conductas adictivas también incrementan el riesgo (3).

Una reducción rápida de la dosis o la suspensión brusca puede producir síntomas de retirada que consisten en cefalea, dolores musculares, ansiedad acusada, confusión, irritabilidad, leve disforia e insomnio. En los casos graves puede aparecer despersonalización, hiperacusia, hormigueo y calambres musculares y abdominales, vómitos, sudoración, temblor, intolerancia a la luz y al contacto físico, alucinaciones o convulsiones (4).

En el momento de la intervención, la paciente tenía una vida estable con proyecto de futuro, no tenía ninguna enfermedad mental, no tomaba otros medicamentos ni tenía adicción a drogas. Quería interrumpir el consumo de alprazolam.

De acuerdo con el médico, se decidió realizar un reajuste de dosis y establecer un plan de reducción paulatina de la dosis, preparando en el laboratorio de la farmacia las cápsulas necesarias para ello a partir de comprimidos debido a la situación de la paciente (5). Se utilizó como referencia el protocolo de la Universidad de Newcastle, en Reino Unido (6), que establece primero un cambio a dosis equivalente de BZD de acción larga (diazepam), seguida de reducciones del $10 \%$ de la dosis en las primeras fases y del $25 \%$ en las últimas. Los protocolos establecen una dosis final de $1 \mathrm{mg}$, pero de acuerdo con el médico, fijamos la dosis final en $0,5 \mathrm{mg}$ de diazepam. La pauta inicial puede verse en la tabla 1.

Este trabajo fue presentado en el VIII Congreso Nacional de Farmacéuticos Comunitarios. Alicante 2018.

Recibido: 27/11/2019

Aceptado: 20/2/2020

Disponible online: $11 / 5 / 2020$
Financiación: ninguna.

Conflicto de intereses: ninguno.

Cite este artículo como: Martín A, Lozano M, Ferrer E. Deshabituación de benzodiazepinas en un paciente preparando cápsulas de diazepam en dosis decrecientes. Farmacéuticos Comunitarios. 2020 May 11;12(2):26-29. doi:10.33620/ FC.2173-9218.(2020/Vol12).002.05

Correspondencia: Ana Martín Ruiz (ana@farmalozanobosch.es). 
Tabla 1 Pauta inicial propuesta

\begin{tabular}{|c|c|c|c|c|c|c|}
\hline & & Mañana & Mediodía & Tarde & Noche & DDED mg \\
\hline Dosis inicial & Fecha inicio/Fin & Alprazolam 0,25 mg & & & Alprazolam $0,25 \mathrm{mg}$ & 10 \\
\hline Fase 1 & & Alprazolam 0,25 mg & & & Alprazolam 0,25 mg & \multirow{2}{*}{10} \\
\hline (2 semanas) & & & & & & \\
\hline Fase 2 & & Alprazolam 0,25 mg & & & Diazepam 5 mg & \multirow{2}{*}{10} \\
\hline (2 semanas) & & & & & & \\
\hline Fase 3 & & Diazepam 5 mg & & & Diazepam 5 mg & \multirow{2}{*}{10} \\
\hline (2 semanas) & & & & & & \\
\hline Fase 4 & & Diazepam 4 mg & & & Diazepam 5 mg & \multirow{2}{*}{9} \\
\hline (1 semana) & & & & & & \\
\hline Fase 5 & & Diazepam 3 mg & & & Diazepam 5 mg & \multirow{2}{*}{8} \\
\hline (1-2 semanas) & & & & & & \\
\hline Fase 6 & & Diazepam 2 mg & & & Diazepam 5 mg & \multirow{2}{*}{7} \\
\hline (1-2 semanas) & & & & & & \\
\hline Fase 7 & & Diazepam $1 \mathrm{mg}$ & & & Diazepam 5 mg & \multirow{2}{*}{6} \\
\hline (2 semanas) & & & & & & \\
\hline Fase 8 & & Diazepam 0,5 mg & & & Diazepam 5 mg & \multirow{2}{*}{5,5} \\
\hline (1-2 semanas) & & & & & & \\
\hline Fase 9 & & & & & Diazepam 5 mg & \multirow{2}{*}{5} \\
\hline (1-2 semanas) & & & & & & \\
\hline Fase 10 & & & & & Diazepam 4 mg & \multirow{2}{*}{4} \\
\hline (1-2 semanas) & & & & & & \\
\hline Fase 11 & & & & & Diazepam 3 mg & \multirow{2}{*}{3} \\
\hline (1-2 semanas) & & & & & & \\
\hline Fase 12 & & & & & Diazepam 2,5 mg & \multirow{2}{*}{2,5} \\
\hline (1-2 semanas) & & & & & & \\
\hline Fase 13 & & & & & Diazepam 2 mg & \multirow{2}{*}{2} \\
\hline (1 semana) & & & & & & \\
\hline Fase 14 & & & & & Diazepam 1,5 mg & \multirow{2}{*}{1,5} \\
\hline (1 semana) & & & & & & \\
\hline Fase 15 & & & & & Diazepam $1 \mathrm{mg}$ & \multirow{2}{*}{1} \\
\hline (1 semana) & & & & & & \\
\hline Fase 16 & & & & & Diazepam 0,5 mg & \multirow{2}{*}{0,5} \\
\hline (1 semana) & & & & & & \\
\hline Fase 17 & & & & & Placebo & \multirow{2}{*}{0} \\
\hline (1 semana) & & & & & & \\
\hline Fase 18 & & & & & Placebo & \multirow{2}{*}{0} \\
\hline (1 semana) & & & & & & \\
\hline
\end{tabular}

DDED: dosis diaria equivalente de diazepam. 
Tabla 2 Peso de los diferentes comprimidos y cápsulas utilizados en la prueba. Media y desviación estándar

\begin{tabular}{|c|c|c|}
\hline$N^{\circ}$ & Partidos & Cápsulas \\
\hline 1 & 0,137 & 0,124 \\
\hline 2 & 0,106 & 0,123 \\
\hline 3 & 0,139 & 0,111 \\
\hline 4 & 0,141 & 0,125 \\
\hline 5 & 0,099 & 0,115 \\
\hline 6 & 0,142 & 0,112 \\
\hline 7 & 0,142 & 0,109 \\
\hline 8 & 0,113 & 0,124 \\
\hline 9 & 0,106 & 0,123 \\
\hline 10 & 0,137 & 0,121 \\
\hline 11 & 0,106 & 0,111 \\
\hline 12 & 0,100 & 0,120 \\
\hline 13 & 0,124 & 0,120 \\
\hline 14 & 0,119 & 0,117 \\
\hline 15 & 0,100 & 0,122 \\
\hline 16 & 0,100 & 0,118 \\
\hline 17 & 0,139 & 0,120 \\
\hline 18 & 0,142 & 0,120 \\
\hline 19 & 0,131 & 0,117 \\
\hline 20 & 0,146 & 0,124 \\
\hline
\end{tabular}

\begin{tabular}{|c|c|c|}
\hline No datos & 20 & 20 \\
\hline Media $m$ & 0,123 & 0,119 \\
\hline Desviación s & 0,01782 & 0,00503 \\
\hline
\end{tabular}

Según los protocolos, cada fase puede durar una o dos semanas. Si la paciente no tolerara bien una reducción, la fase puede prolongarse el tiempo necesario, pero nunca se vuelve a subir la dosis. Por ello, no es posible conocer a priori la duración del tratamiento, aunque haya un plan establecido, ya que es la paciente la que marca la duración de cada período.

\section{Intervención}

Todos los procedimientos consultados (6) ajustaban la dosis raspando y pesando los comprimidos uno a uno hasta el peso indicado. Aunque en principio consideramos este método, lo descartamos porque nos pareció más lento y por no disponer del procedimiento de elaboración, mientras que para la encapsulación (PN/L/FF/001 del Formulario Nacional y PNT11-4 MICOF GUIA FORMULACION MAGISTRAL) por diluciones geométricas (PNT11-2 MICOF GUIA

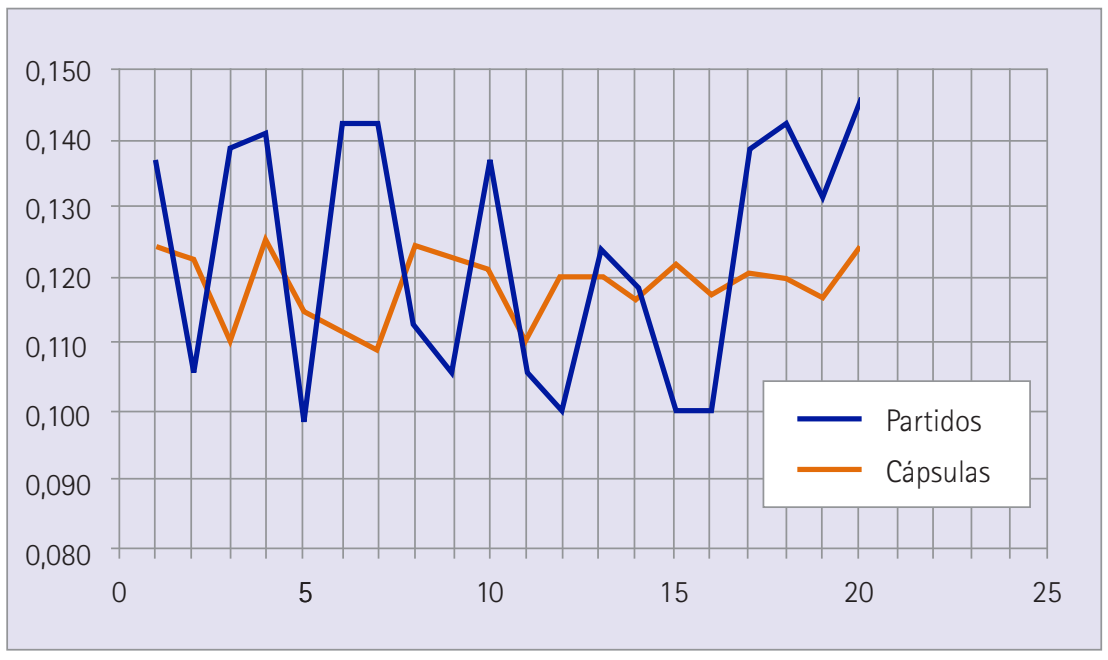

Figura 1 Peso de los diferentes comprimidos y cápsulas utilizados en la prueba

FORMULACION MAGISTRAL) sí que disponíamos de procedimientos normalizados. Como se puede observar en los datos (tabla 2 y figura 1), comprobamos que la partición de comprimidos presentaba una desviación respecto a la media mayor que las cápsulas, en cuanto al contenido de principio activo. Nosotros preferimos preparar cápsulas con reducción paulatina de dosis utilizando los protocolos de reducción lenta de la profesora Heather Asthon, de la Universidad de Newcastle (1), y en el caso de que aparecieran síntomas de abstinencia se replantearía la dosificación sin alterar la DDED (dosis diaria equivalente de diazepam). Se plantearon reducciones de dosis en promedios del $10 \%$ en las fases iniciales y del $25 \%$ en las fases de toma única, se consultó con la paciente y el médico y tras su conformidad comenzamos a elaborar las cápsulas. Para ello trituramos comprimidos de diazepam Leo y los diluimos con excipiente $\mathrm{N}^{\circ} 1 \mathrm{del}$ Formulario Nacional, utilizando riboflavina como indicador de dilución. Para preparar las cápsulas seguimos el PNT adecuado.

Debido a la importancia de la dosis por toma en el proceso de deshabituación, calculamos la desviación estándar del contenido en principio activo de 20 comprimidos partidos por la paciente $(s=0,018)$ y de 20 cápsulas hechas en el laboratorio $(s=0,005), y$ comprobamos que según se aprecia en el gráfico, nuestro método es más exacto.

Respecto al tiempo, se reparte en preparar la mezcla de polvos y llenar las cápsulas. Como el contenido en principio activo siempre es decreciente, el trabajo consiste en diluir la mezcla de polvos inicial. El tiempo empleado para preparar, encapsular y envasar las necesidades semanales pueden variar entre 3 y 5 minutos.

En cuanto al coste, el importe de cada mes lo establecimos en $20 €$. El precio incluye tanto la preparación de medicamento como los trabajos necesarios para el cumplimiento del programa de deshabituación, como son el recálculo de dosis, prospectos e información al paciente y coordinación con el médico.

Desconocemos el tiempo necesario para preparar las dosis mediante raspado de comprimidos y, en consecuencia, el posible importe del servicio.

\section{Resultado/Seguimiento}

La paciente retiraba de la farmacia semanalmente su medicación. También, antes de preparar las cápsulas, consultábamos con ella si manteníamos o avanzábamos siguiendo el plan propuesto porque era decisión suya establecer la duración de la fase, siempre sin alterar la DDED. La paciente respondió perfectamente al tratamiento y decidió que se acelerara. Se habían previsto inicialmente 19 fases con una duración total de 38 semanas, pero se pudo reducir a 15 fases en 18 semanas.

Cuando se suspendió definitivamente la BZD se cambió a fitoterapia para que fuera todavía menos brusca la interrupción. Primero preparando cápsulas de Pasiflora con Espino albar y posteriormente con comprimidos de Aquilea sueño ${ }^{\circledR}$ (Amapola de California, Pasiflora, Valeriana y Melatonina $1,95 \mathrm{mg}$ ). 


\section{Comentario final/ Conclusiones}

El procedimiento ha sido plenamente satisfactorio para todas las partes. La paciente ha sido muy colaboradora en todo momento. La comunicación paciente-farmacia-médico es fundamental para llevar a buen término un tratamiento de deshabituación. No sólo porque es el médico el que debe generar las recetas necesarias para poder utilizar el diazepam, sino porque facilita cualquier consulta sobre cambios de pauta. Al mismo tiempo, el paciente está más tranquilo sabiendo que médico y farmacia están colaborando.

La toma de decisiones compartida con el paciente, antes y durante el proceso, le anima a asumir responsabilidades y a cumplir con el tratamiento, el cual debe individualizarse para cada caso según su personalidad, condiciones de vida y entorno socio-ambiental.
Este sistema de reducción gradual de dosis es aplicable a otros principios activos como corticoides, antidepresivos, antipsicóticos, antiparkinsonianos, etc. También permite la adecuación de dosis a pacientes de muy bajo peso o extremadamente debilitados que necesiten dosis inferiores a las comercializadas.

\section{Referencias bibliográficas}

1. Agencia Española de medicamentos y productos sanitarios. [Sede web]. Madrid; [último acceso 25/11/2019]. Utilización de medicamentos ansiolíticos e hipnóticos en España durante el periodo 2010-2018. https://www.aemps. gob.es/medicamentos-de-uso-humano/observatorio-de-uso-de-medicamentos/informes-ansioliticos-hipnoticos-espana-2010-2018/

2. Cámara R, Cosín A, Dago A, Gutiérrez P, Salar L, Solá N. Programa D-VALOR, análisis de registros de dispensación. Benzodiazepinas. Pharm Care Esp. 2013; 15(4): 140-146.
3. SOVAMFiC. Societat Valenciana de Medicina Familiar y Comunitària. [Sede web]. Valencia; [último acceso 25/11/2019]. Fichas de consulta rápida de deshabituación a benzodiazepinas. http://www.svmfyc.org/files/Fichas_ Consulta_Rapida/2016_DESHABITUACION_A_BENZODIAZEPINAS.pdf

4. Agencia Española de medicamentos y productos sanitarios. [Sede web]. Madrid; [último acceso 25/11/2019]. Ficha técnica Trankimazin. https://cima. aemps.es/cima/pdfs/es/ft/64079/FichaTecnica_64079.html.pdf

5. Documento BOE-A-2009-12002. RD 1015/2009, de 19 de junio, por el que se regula la disponibilidad de medicamentos en situaciones especiales. («BOE» núm. 174, de 20 de julio de 2009, páginas 60904 a 60913) https://www.boe.es/ buscar/doc.php?id=BOE-A-2009-12002

6. Newcastle University. The Institute of Neuroscience. [Sede web] Newcastle: Profesor C.H. Ashton; [último acceso 25/11/2019]. Las Benzodiacepinas: cuál es su mecanismo y como suspender la ingestión. https://benzo.org.uk/ espman/ 